\title{
AMBULATORY BLOOD PRESSURE MONITORING INDICATORS IN MIDDLE-AGED AND ELDERLY HYPERTENSIVE PATIENTS UNDER COMBINED ANTIHYPERTENSIVE THERAPY
}

\author{
V. S. Pasko
}

State institution of science «Research and practical center of preventive and clinical medicine» State administrative department, Kyiv, Ukraine

\section{Summary}

It is known that arterial hypertension affects $30-35 \%$ of adult population of industrialized countries, and its prevalence reaches $40-50 \%$ among the elderly. In recent years, the issue of optimizing of treatment of hypertension in elderly people has been discussed such as choice of effective antihypertensive drugs with the least number of side effects, prevention of complications, reduced mortality, improvement of quality of life.

The aim of the study was to substantiate the antihypertensive efficacy of the combination of enalapril and amlodipine in middle-aged and elderly hypertensive patients.

Material and methods. Indices of ambulatory blood pressure monitoring were identified in 57 middle-aged patients (45-59 years) (group I) and 43 elderly patients (60-74 years) (group II), who underwent two-week in-patient treatment. All patients received the combined antihypertensive therapy (enalapril $10 \mathrm{mg}$ twice daily and amlodipine $5 \mathrm{mg}$ daily) for 2 weeks. And they were divided into 2 groups of 24 hours day's profile of blood pressure: dippers and non-dippers.

Results. The combination of enalapril and amlodipine in elderly patients reduced the average daily diastolic blood pressure by $20,3 \%(p<0,05)$. Instead, in middle-aged patients there was the decrease in the average daily and the maximal daily systolic blood pressure by $17,3 \%(p<0,001)$ and $7,8 \%$ $(p<0,05)$ and diastolic blood pressure respectively - by 23,2\% $(p<0,001)$ and $11,8 \%(p<0,05)$.

Conclusions. Therefore, combined antihypertensive therapy in the elderly for 14 days was insufficient to improve significantly ambulatory blood pressure monitoring indicators. Obviously, positive changes require longer treatment.

Keywords: arterial hypertension, essential hypertension, blood pressure, ambulatory blood pressure monitoring, combined antihypertensive therapy.

It is known that arterial hypertension affects $30-35 \%$ of adult population of industrialized countries, and its prevalence reaches $40-50 \%$ among the elderly. In recent years, the issue of optimizing of treatment of hypertension in elderly people has been discussed such as choice of effective antihypertensive drugs with the least number of side effects, prevention of complications, reduced mortality, improvement of quality of life [1-9].

The objective of the study. To substantiate the antihypertensive efficacy of the combination of enalapril and amlodipine in middle-aged and elderly hypertensive patients.

Material and methods. Indices of ambulatory blood pressure monitoring (ABPM) were identified in 57 middle-aged patients (45-59 years) (group I) and 43 elderly patients (60-74 years) (group II), who underwent two-week in-patient treatment. All patients received the combined antihypertensive therapy (enalapril $10 \mathrm{mg}$ twice daily and amlodipine $5 \mathrm{mg}$ daily) for 2 weeks. And they were divided into 2 groups of 24 hours day's profile of blood pressure (BP): dippers if reducing of BP was enough at night (daily index (DI) - 10-20\%) and non-dippers with a little decrease in BP at night (DI-0-10\%).

Indicators of $\mathrm{ABPM}$ were determined using a portable recorder ABPM-04 (company «Meditech», Hungary) according to a standard protocol [10]. Measurements of BP and some other parameters were performed every 15 minutes during daily activity (from 6 to 22 hours) and every 30 minutes during night sleep (from 22 to 6 hours). The initial BP measurement was performed on both hands 
of a patient and subsequent registration was carried on the arm with higher initial pressure values. We determined and analyzed the following indices: the average daily systolic BP (SBP) (mm Hg), the average daily diastolic BP (DBP) $(\mathrm{mm} \mathrm{Hg})$, the average $\mathrm{BP}(\mathrm{mm} \mathrm{Hg})$, the maximal daily SBP (mm Hg), the maximal daily DBP $(\mathrm{mm} \mathrm{Hg})$, the average daily heart rate (HR) (/min.), DI of SBP (\%), DI of DBP $(\%)$, DI of the average BP $(\%)$.

The results of the surveys were processed by the methods of variation statistics by determining for each variation series the arithmetic mean «M» and the standard deviation $\sigma$. Statistical data processing was performed using the Microsoft Excel package.

Results and discussion. Against the background of the combined antihypertensive therapy with enal- april and amlodipine the results of ABPM in the middle-aged (Table 1) showed the significant decrease in the average daily SBP by $17,3 \%$ from $156,5 \pm 12,1 \mathrm{~mm} \mathrm{Hg}$ to $129,5 \pm 8,8 \mathrm{~mm} \mathrm{Hg} ; \mathrm{p}<0,001$; the maximal SBP by 7,8 (from $176,5 \pm 23,5 \mathrm{~mm} \mathrm{Hg}$ to $162,7 \pm 15,3 \mathrm{~mm} \mathrm{Hg}$; $\mathrm{p}<0,05$; the average daily DBP by $23,2 \%$ (from $99,9 \pm 6,6 \mathrm{~mm} \mathrm{Hg}$ to $76,7 \pm 6,7 \mathrm{~mm} \mathrm{Hg} ; \mathrm{p}<0,001)$; the maximal DBP by $11,8 \%$ (from 115,6 $\pm 21,0 \mathrm{~mm} \mathrm{Hg}$ to 102,5 $\pm 10,2 \mathrm{~mm} \mathrm{Hg}$; $\mathrm{p}<0,05$ ). The greatest decrease in the average daily DBP (by 23,2\%) and the average daily SBP (by 17,3\%) to a lesser extent the maximal SBP and DBP (7,8\% and $11,8 \%$ respectively).

In elderly hypertensive patients significant changes affected only the average daily DBP, there was a decrease of $20,3 \%(\mathrm{p}<0,05)$ (from $92,5 \pm 1,3 \mathrm{~mm} \mathrm{Hg}$ to $73,7 \pm 6,8 \mathrm{~mm}$ $\mathrm{Hg})$.

\section{Ambulatory blood pressure monitoring indicators in middle-aged and elderly hypertensive patients under} combined antihypertensive therapy $(\mathrm{M} \pm \sigma)$

\begin{tabular}{|c|c|c|c|c|c|}
\hline \multirow{3}{*}{ The indices } & \multicolumn{4}{|c|}{ The distribution of the surveyed groups } & \multirow{3}{*}{$\mathbf{p}$} \\
\hline & \multicolumn{2}{|c|}{ Group I } & \multicolumn{2}{|c|}{ Group II } & \\
\hline & $\begin{array}{c}\text { Before the } \\
\text { therapy } \\
(n=57)\end{array}$ & $\begin{array}{c}\text { After the } \\
\text { therapy } \\
(n=30)\end{array}$ & $\begin{array}{c}\text { Before the } \\
\text { therapy } \\
(n=43)\end{array}$ & $\begin{array}{c}\text { After the } \\
\text { therapy } \\
(n=16)\end{array}$ & \\
\hline $\begin{array}{l}\text { The average daily SBP } \\
(\mathrm{mm} \mathrm{Hg})\end{array}$ & $156,5 \pm 12,1$ & $129,5 \pm 8,8$ & $150,5 \pm 12,6$ & $127,0 \pm 11,2$ & $\begin{array}{c}\mathbf{p}_{1}<0,001 \\
\mathbf{p}_{2}>0,05\end{array}$ \\
\hline $\begin{array}{l}\text { The average daily DBP } \\
(\mathrm{mm} \mathrm{Hg})\end{array}$ & $99,9 \pm 6,6$ & $76,7 \pm 6,7$ & $92,5 \pm 1,3$ & $73,7 \pm 6,8$ & $\begin{array}{c}\mathbf{p}_{1}<0,001 \\
\mathbf{p}_{2}<0,05\end{array}$ \\
\hline $\begin{array}{l}\text { The average BP } \\
(\mathrm{mm} \mathrm{Hg})\end{array}$ & $116,2 \pm 10,0$ & $97,4 \pm 12,2$ & $103,3 \pm 10,9$ & $91,4 \pm 6,9$ & $\begin{array}{l}\mathbf{p}_{1}>0,05 \\
\mathbf{p}_{2}>0,05\end{array}$ \\
\hline $\begin{array}{l}\text { The maximal daily SBP } \\
(\mathrm{mm} \mathrm{Hg})\end{array}$ & $176,5 \pm 23,5$ & $162,7 \pm 15,3$ & $169,3 \pm 25,1$ & $163,1 \pm 17,0$ & $\begin{array}{l}\mathbf{p}_{1}<0,05 \\
\mathbf{p}_{2}>0,05\end{array}$ \\
\hline $\begin{array}{l}\text { The maximal daily DBP } \\
(\mathrm{mm} \mathrm{Hg})\end{array}$ & $115,6 \pm 21,0$ & $102,5 \pm 10,2$ & $100,4 \pm 18,2$ & $99,6 \pm 16,1$ & $\begin{array}{l}\mathbf{p}_{1}<0,05 \\
\mathbf{p}_{2}>0,05\end{array}$ \\
\hline The average daily HR (/min.) & $65,5 \pm 8,4$ & $66,9 \pm 9,2$ & $63,0 \pm 9,1$ & $63,7 \pm 9,2$ & $\begin{array}{l}\mathbf{p}_{1}>0,05 \\
\mathbf{p}_{2}>0,05\end{array}$ \\
\hline DI of SBP $(\%)$ & $9,6 \pm 7,4$ & $7,1 \pm 8,8$ & $8,9 \pm 5,5$ & $7,1 \pm 8,1$ & $\begin{array}{l}\mathbf{p}_{1}>0,05 \\
\mathbf{p}_{2}>0,05 \\
\end{array}$ \\
\hline DI of DBP $(\%)$ & $13,8 \pm 8,1$ & $10,6 \pm 9,8$ & $14,1 \pm 7,1$ & $10,6 \pm 12,2$ & $\begin{array}{l}\mathbf{p}_{1}>0,05 \\
\mathbf{p}_{2}>0,05 \\
\end{array}$ \\
\hline DI of the average BP $(\%)$ & $12,0 \pm 7,7$ & $6,1 \pm 13,2$ & $11,3 \pm 6,1$ & $8,8 \pm 9,6$ & $\begin{array}{l}\mathbf{p}_{1}>0,05 \\
\mathbf{p}_{2}>0,05\end{array}$ \\
\hline
\end{tabular}

Remark: p1 - statistical significance of difference between indicators of patients of group I, p2 - statistical significance of difference between indicators of patients of group II.

Given the degree of decrease in nocturnal blood pressure in middle-aged hypertensive patients in the dipper group ABPM values decreased significantly (Table 2): the average daily SBP by $17,7 \%$ (from $155,9 \pm 12,0 \mathrm{~mm} \mathrm{Hg}$ to
$128,3 \pm 10,2 \mathrm{~mm} \mathrm{Hg}(\mathrm{p}<0,001))$; the average daily DBP by $21,6 \%$ (from $97,3 \pm 6,1 \mathrm{~mm} \mathrm{Hg}$ to $76,3 \pm 6,8 \mathrm{~mm} \mathrm{Hg}$; $\mathrm{p}<0,001$ and the average BP by $12,8 \%$ (from 110,0 $\pm 3,6 \mathrm{~mm}$ $\mathrm{Hg}$ to $95,9 \pm 6,5 \mathrm{~mm} \mathrm{Hg}$; $<0,01)$. 
Ambulatory blood pressure monitoring indicators in middle-aged hypertensive patients of the dipper and the non-dipper group under combined antihypertensive therapy with enalapril and amlodipine $(\mathbf{M} \pm \sigma)$

\begin{tabular}{|c|c|c|c|c|c|}
\hline \multirow{3}{*}{ The indices } & \multicolumn{4}{|c|}{ The distribution of the surveyed groups } & \multirow{3}{*}{$\mathrm{p}$} \\
\hline & \multicolumn{2}{|c|}{ The dipper group } & \multicolumn{2}{|c|}{ The non-dipper group } & \\
\hline & $\begin{array}{l}\text { Before the therapy } \\
\qquad(n=32)\end{array}$ & $\begin{array}{l}\text { After the } \\
\text { therapy } \\
(n=15)\end{array}$ & $\begin{array}{l}\text { Before the } \\
\text { therapy } \\
(n=25)\end{array}$ & $\begin{array}{l}\text { After the } \\
\text { therapy } \\
(n=15)\end{array}$ & \\
\hline $\begin{array}{l}\text { The average daily SBP } \\
(\mathrm{mm} \mathrm{Hg})\end{array}$ & $155,9 \pm 12,0$ & $128,3 \pm 10,2$ & $157,1 \pm 12,9$ & $131,3 \pm 8,5$ & $\begin{array}{c}\mathbf{p}_{1}<0,001 \\
\mathbf{p}_{2}<0,01\end{array}$ \\
\hline $\begin{array}{l}\text { The average daily DBP } \\
(\mathrm{mm} \mathrm{Hg})\end{array}$ & $97,3 \pm 6,1$ & $76,3 \pm 6,8$ & $106,0 \pm 1,0$ & $77,1 \pm 6,8$ & $\begin{array}{l}\mathbf{p}_{1}<0,001 \\
\mathbf{p}_{2}<0,05\end{array}$ \\
\hline $\begin{array}{l}\text { The average BP } \\
\quad(\mathrm{mm} \mathrm{Hg})\end{array}$ & $110,0 \pm 3,6$ & $95,9 \pm 6,5$ & $124,3 \pm 6,8$ & $98,8 \pm 9,9$ & $\begin{array}{l}\mathbf{p}_{1}<0,01 \\
\mathbf{p}_{2}>0,05\end{array}$ \\
\hline $\begin{array}{l}\text { The maximal daily SBP } \\
(\mathrm{mm} \mathrm{Hg})\end{array}$ & $176,3 \pm 23,5$ & $165,9 \pm 15,2$ & $176,9 \pm 24,0$ & $159,5 \pm 15,3$ & $\begin{array}{l}\mathbf{p}_{1}>0,05 \\
\mathbf{p}_{2}>0,05\end{array}$ \\
\hline $\begin{array}{l}\text { The maximal daily DBP } \\
(\mathrm{mm} \mathrm{Hg})\end{array}$ & $117,0 \pm 19,2$ & $107,3 \pm 8,0$ & $113,8 \pm 23,3$ & $97,8 \pm 10,3$ & $\begin{array}{l}\mathbf{p}_{1}>0,05 \\
\mathbf{p}_{2}<0,05\end{array}$ \\
\hline The average daily HR (/min.) & $66,5 \pm 7,3$ & $66,2 \pm 9,4$ & $64,2 \pm 9,8$ & $67,5 \pm 9,33$ & $\begin{array}{l}\mathbf{p}_{1}>0,05 \\
\mathbf{p}_{2}>0,05\end{array}$ \\
\hline DI of SBP $(\%)$ & $14,7 \pm 4,3$ & $13,4 \pm 2,8$ & $3,0 \pm 5,0$ & $0,8 \pm 8,3$ & $\begin{array}{l}\mathbf{p}_{1}>0,05 \\
\mathbf{p}_{2}>0,05\end{array}$ \\
\hline DI of DBP $(\%)$ & $18,8 \pm 5,1$ & $16,5 \pm 4,6$ & $7,5 \pm 6,5$ & $4,6 \pm 10,1$ & $\begin{array}{l}\mathbf{p}_{1}>0,05 \\
\mathbf{p}_{2}>0,05\end{array}$ \\
\hline DI of the average BP (\%) & $17,1 \pm 4,5$ & $15,1 \pm 3,5$ & $5,4 \pm 5,7$ & $3,3 \pm 9,2$ & $\begin{array}{l}\mathbf{p}_{1}>0,05 \\
\mathbf{p}_{2}>0,05\end{array}$ \\
\hline
\end{tabular}

Remark: p1-statistical significance of difference between before the therapy and after the therapy in the dipper group, p2-statistical significance of difference between before the therapy and after the therapy in the non-dipper group.

In middle-aged hypertensive patients of the non-dipper group like patients in the dipper group the average daily SBP decreased (from $157,1 \pm 12,9 \mathrm{~mm} \mathrm{Hg}$ to $131,3 \pm 8,5 \mathrm{~mm}$ $\mathrm{Hg} ; \mathrm{p}_{2}<0,01$; the average daily DBP from $106,0 \pm 1,0 \mathrm{~mm}$ $\mathrm{Hg}$ to $77,1 \pm 6,8 \mathrm{~mm} \mathrm{Hg} ; \mathrm{p}<0,05)$ by $16,4 \%$ and $27,3 \%$ respectively. The maximal daily DBP decreased by $14,1 \%$ (from 113,8 $\pm 23,3 \mathrm{~mm} \mathrm{Hg}$ to $97,8 \pm 10,3 \mathrm{~mm} \mathrm{Hg} ; \mathrm{p}<0,05$ ).

Conclusions. The combination of enalapril and amlodipine in elderly patients reduced the average daily
DBP by 20,3\% ( $\mathrm{p}<0,05)$. Instead, in middle-aged patients there was the decrease in the average daily and the maximal daily SBP by $17,3 \%(\mathrm{p}<0,001)$ and $7,8 \%$ $(p<0,05)$ and DBP respectively - by $23,2 \%(p<0,001)$ and $11,8 \%(\mathrm{p}<0,05)$.

Therefore, combined antihypertensive therapy in the elderly for 14 days was insufficient to improve significantly ABPM indicators. Obviously, positive changes require longer treatment.

\section{ЛITEPATУРA}

1. Біканова I.I., Вікторов О. П. Використання еналаприлу: сучасні проблеми. Новости медицины и фармации. 2007. № 5 (209). С. 28.

2. Билецкий С.В., Петринич О. А., Казанцева Т. В. Роль антагонистов кальция в лечении ар- териальной гипертензии. Внутрішня медицина. 2008. № 1 (7). С. 50-53.

3. Бичко М. В. Антагоністи кальцію в лікуванні хворих на артеріальну гіпертензію, асоційовану із ішемічною хворобою серця. Ужгород: Ліра. 2012. 376 с. 
4. Жарінов О.Й., Децик О. Б., Рогуцька О. М. Ефективність амлодипіну у зниженні систолічного артеріального тиску: відкрите оглядове дослідження. Український кардіологічний журнал. 2007. № 2. С. 39-44.

5. Житникова Л. М. Комбинированная терапия артериальной гипертонии: эффективно и удобно. Русский медицинский журнал. 2011. Том 19. № 2. С. 1-6.

6. Иванова Л. Н. Амлодипин в терапии больных артериальной гипертензией. Укр. мед. альманах. 2007. Том 47. № 7. С. 27-28.

7. Лопатин Ю. М. Амлодипин как компонент комбинированной антигипертензионной терапии: что определяет выбор препарата? «Consilium medicum». 2008. Экстра спец. выпуск. С. 6-11.

8. Маколкин В. И. Антагонисты кальция - препараты выбора при лечении артериальной гипертензии. Внутрішня медицина. 2007. № 5. С. 98-100.

9. Остроумова О.Д., Шапошник И. И., Ролик Н. Л., Поликарпов В.А. Возможности комбинированной терапии ингибиторами АПФ и дигидропиридиновыми антагонисатми кальция. Український медичний часопис. 2006. № 1 (51). С. 41-48.

10.Сіренко Ю. М., Радченко Г. Д. Значення добового моніторування артеріального тиску для діагностики і лікування артеріальної гіпертензіі. К. 2001. 27 с.

\section{REFERENCES}

1. Bikanova, I.I., Viktorov, O.P. (2007). Vikoristannya enalaprilu: suchasni problemy [The use of enalapril: current problems]. Novosti mediciny i farmacii, 5 (209), 28.

2. Bileckij, S.V., Petrinich, O.A., Kazanceva, T.V. (2008). Rol' antagonistov kal'ciya v lechenii arterial'noj gipertenzii [The role of calcium antagonists in the treatment of hypertension]. Internal Medicine, 1 (7), 50-53.

3. Bichko, M.V. (2012). Antagonisti kal'ciyu v likuvanni hvorih na arterial'nu gipertenziyu, asocijovanu iz ishemichnoyu hvoroboyu sercya [Calcium antagonists in the treatment of patients with hypertension associated with coronary artery disease]. Uzhgorod: Lira, 376.

4. ZHarinov, O.J., Decik, O.B., Roguc'ka, O.M. (2007). Efektivnist' amlodipinu u znizhenni sistolichnogo arterial'nogo tisku: vidkrite oglyadove doslidzhennya [Efficacy of amlodipine in lowering of systolic blood pressure: an open-label study]. Ukrainian Journal of Cardiology, 2, 39-44.

5. ZHitnikova, L.M. (2011). Kombinirovannaya terapiya arterial'noj gipertonii: effektivno i udobno [Combination therapy of arterial hypertension: effective and convenient]. Russian medical journal, 19, 2, 1-6.

6. Ivanova, L.N. (2007). Amlodipin v terapii bol'nyh arterial'noj gipertenziej [Amlodipine in the treatment of patients with hypertension]. Ukrainian Medical Almanac, 47, 7, 27-28.

7. Lopatin, YU.M. (2008). Amlodipin kak komponent kombinirovannoj antigipertenzionnoj terapii: chto opredelyaet vybor preparata? [Amlodipine as a component of combination antihypertensive therapy: what determines the choice of drug?]. Consilium medicum, 6-11.

8. Makolkin, V.I. (2007). Antagonisty kal'ciya - preparaty vybora pri lechenii arterial'noj gipertenzii [Calcium antagonists are the drugs of choice in the treatment of hypertension]. Internal Medicine, 5, 98-100.

9. Ostroumova, O.D., SHaposhnik, I.I., Rolik, N.L., Polikarpov, V.A. (2006). Vozmozhnosti kombinirovannoj terapii ingibitorami APF i digidropiridinovymi antagonisatmi kal'ciya [Possibilities of combination therapy with ACE inhibitors and calcium dihydropyridine antagonists]. Ukrainian medical journal, 1 (51), 41-48.

10.Sirenko, YU. M., Radchenko, H.D. (2001). Znachennya dobovoho monitoruvannya arterialnoho tysku dlya diahnostyky i likuvannya arterialnoyi hipertenziyi [Value of ambulatory blood pressure monitoring for diagnosis and treatment of arterial hypertension]. K., 27. 


\section{Резюме}

\section{ПОКАЗНИКИ ДОБОВОГО МОНІТОРУВАННЯ АРТЕРІАЛЬНОГО ТИСКУ У ХВОРИХ НА ГІПЕРТОНІЧНУ ХВОРОБУ СЕРЕДНЬОГО ТА ПОХИЛОГО ВІКУ НА ТЛІ КОМБІНОВАНОГО АНТИГІПЕРТЕНЗИВНОГО ЛІКУВАННЯ ЕНАЛАПРИЛОМ ТА АМЛОДИПІНОМ \\ В. С. Пасько}

Державна наукова установа «Науково-практичний центр профілактичної та клінічної медицини» Державного управління справами, м.Київ, Україна

Як відомо, артеріальною гіпертензією страждають 30-35\% дорослого населення індустріально розвинених країн, а серед осіб похилого віку поширеність ї̈ сягає 40-50\%. Останніми роками дискутується питання щодо оптимізації лікування артеріальної гіпертензії в осіб похилого віку. Аонедавна суперечливі питання стосувалися лікування хворих похилого віку на артеріальну гіпертензію, вибору ефективних антигіпертензивних препаратів з найменшою кількістю побічних дій, з попередженням ускладнень артеріальної гіпертензії, зменшенням смертності, покращенням якості життя.

Мета - обгрунтування антигіпертензивної ефективності комбінації еналаприлу і амлодипіну у хворих на гіпертонічну хворобу II стадії похилого та середнього віку.

Матеріал і методи. Показники добового моніторингу артеріального тиску визначено в 57 пацієнтів середнього (45-59 років) віку (група I) і 43 хворих похилого (60-74 роки) віку (група II), які перебували на стаціонарному двотижневому лікуванні. Аікування гіпертонічної хвороби проводилось протягом 14 діб. Використовували комбінацію препарату еналаприлу у дозі 10 мг двічі на добу та амлодипіну у дозі 5 мг на добу. В обстежених середнього і похилого віку виділяли 2 групи добового профілю артеріального тиску: «dipper» $\mathrm{i}$ «non-dipper».

Результати. Комбінація еналаприлу і амлодипіну у хворих похилого віку знижувала середньодобовий діастолічний артеріальний тиск на 20,3\% ( $<<0,05)$. Натомість, у хворих середнього віку відмічене зниження середньодобового та максимального систолічного артеріального тиску на $17,3 \%(\mathrm{p}<0,001)$ і $7,8 \%$ $(\mathrm{p}<0,05)$ та відповідно діастолічного артеріального тиску - на 23,2\% (p<0,001) та $11,8 \%(\mathrm{p}<0,05)$.

Висновки. Отже, проведення комбінованої антигіпертензивної терапії у хворих похилого віку протягом 14 діб було недостатнім для значущого покращення показників добового моніторингу артеріального тиску. Вочевидь, позитивні зміни потребують білыш тривалого медикаментозного впливу.

Ключові слова: артеріальна гіпертензія, гіпертонічна хвороба, артеріальний тиск, добовий моніторинг артеріального тиску, комбіноване антигіпертензивне лікування. 


\section{Резюме}

\section{ПОКАЗАТЕЛИ СУТОЧНОГО МОНИТОРИРОВАНИЯ АРТЕРИАЛЬНОГО ДАВЛЕНИЯ У БОЛЬНЫХ ГИПЕРТОНИЧЕСКОЙ БОЛЕЗНЬЮ СРЕДНЕГО И ПОЖИЛОГО ВОЗРАСТА НА ФОНЕ КОМБИНИРОВАННОГО АНТИГИПЕРТЕНЗИВНОГО ЛЕЧЕНИЯ ЭНАЛАПРИЛОМ И АМЛОДИПИНОМ

\author{
B. С. Пасько
}

Государственное научное учреждение «Научно-практический центр профилактической и клинической медицины» Государственного управления делами, г. Киев, Украина

Как известно, артериальной гипертензией страдают 30-35\% взрослого населения индустриально развитых стран, а среди пожилых Аюдей распространенность ее достигает 40-50\%. В последние годы обсужАается вопрос оптимизации лечения артериальной гипертензии у лиц пожилого возраста. Ао недавнего времени спорные вопросы касались лечения больных пожилого возраста с артериальной гипертензией, выбора эффективных антигипертензивных препаратов с самым меньшим количеством побочных действий, с предупреждением осложнений артериальной гипертензии, уменышением смертности, улучшением качества жизни.

Цель - обоснование антигипертензивной эффективности комбинации эналаприла и амлодипина у больных гипертонической болезнью II стадии пожилого и среднего возраста.

Материал и методы. Показатели суточного мониторинга артериального давления определены у 57 пациентов среднего (45-59 лет) возраста (группа I) и 43 больных пожилого (60-74 года) возраста (группа II), которые находились на стационарном двухнедельном лечении. Иечение гипертонической болезни проводилось в течение 14 суток. Использовали комбинацию препарата эналаприла в дозе 10 мг два раза в сутки и амлодипина в дозе 5 мг в сутки. У обследованных среднего и пожилого возраста выделяли 2 группы суточного профиля артериального давления: «dipper» и «non-dipper».

Результаты. Комбинация эналаприла и амлодипина у больных пожилого возраста снижала среднесуточное диастолическое артериальное давление на 20,3\% ( $<<0,05)$. Зато у больных среднего возраста отмечено снижение среднесуточного и максимального систолического артериального давления на $17,3 \%$ $(\mathrm{p}<0,001)$ и $7,8 \%(\mathrm{p}<0,05)$ и в соответствии диастолического артериального давления - на $23,2 \%(\mathrm{p}<0,001)$ и $11,8 \%(\mathrm{p}<0,05)$.

Выводы. Таким образом, проведение комбинированной антигипертензивной терапии у больных пожилого возраста в течение 14 суток было недостаточным для значимого улучшения показателей суточного мониторинга артериального давления. Очевидно, положительные изменения требуют более длительного медикаментозного воздействия.

Ключевие слова: артериальная гипертензия, гипертоническая болезнь, артериальное давление, суточный мониторинг артериального давления, комбинированное антигипертензивное лечение.

Інформація про авторів знаходиться на сайті http://www.cp-medical.com.

Дата надходження до редакції-3.10.2021 p. 